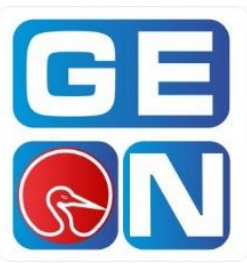

Revista GEON (Gestión, Organizaciones y Negocios.) ISSN: 2346-3910 en línea

revistageon@unillanos.edu.co

Universidad de los Llanos

Colombia

Martínez Muñoz, Enriquei; Hernández-Gracia, Tirso Javierii;

Martínez Muñoz, Bernardino ${ }^{i i i}$; Montesinos Hernández, Joeliv .

Estudio de Mobbing en una institución de educación superior en Hidalgo

Revista GEON, Vol. 6, No. 2, 2019

Pág. 25-36

Disponible en: https://doi.org/10.22579/23463910.153

' https://orcid.org/0000-0001-6418-5292

ii https://orcid.org/0000-0003-0425-0800

iii https://orcid.org/0000-0002-8012-5764

iv https://orcid.org/0000-0002-7296-1666

Esta publicación se encuentra bajo licencia: Creative Commons

Reconocimiento-

NoComercial-

SinObraDerivada

4.0 Internacional

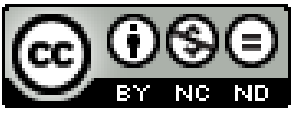

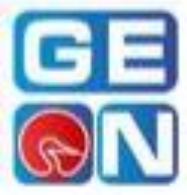

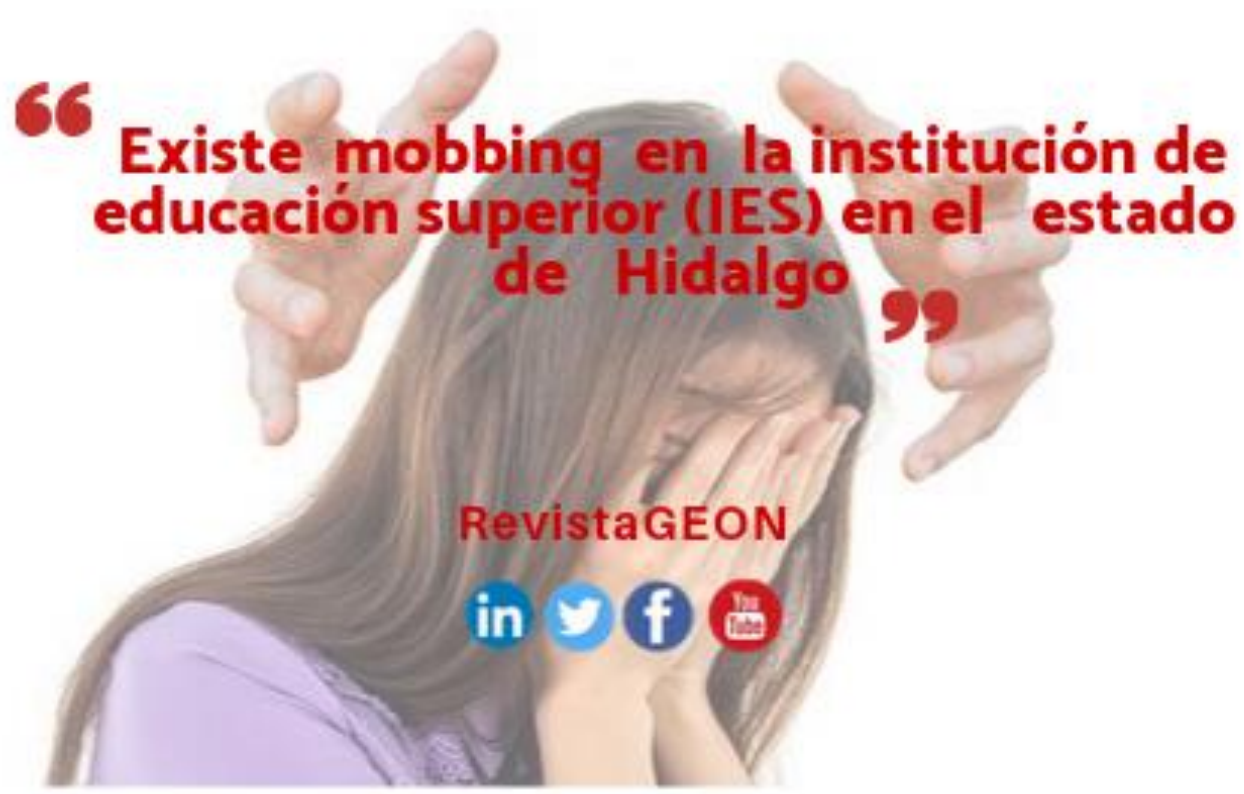

Muñoz, E, Herndindez-Gracia, T., Martinez Murioz, B, a Montesinos Herndindez, I. [2019]. Estudio de Mobbinq en una institución de educación superior en Hidalgo 


\section{Estudio de Mobbing en una institución de educación superior en Hidalgo, México}

\section{Study of Mobbing in a higher education institucion in Hidalgo, México}

\section{Enrique Martínez Muñoz ${ }^{1}$ Tirso Javier Hernández Gracia ${ }^{2}$ Bernardino Martínez, Muñoz, Joel Montesinos Hernández ${ }^{4}$}

Cómo citar este artículo / To reference this article:
Muñoz, E., Hernández-Gracia, T., Martínez Muñoz, B., \& Montesinos Hernández, J. (2019). Estudio de Mobbing en una institución de educación superior en Hidalgo. Revista GEON (Gestión, Organizaciones Y Negocios), 6(2), 25-37. Recuperado a partir de http://revistageon.unillanos.edu.co/index.php/geon/article/view/153
Artículo de investigación:

Fecha de recepción: $2019 / 04 / 20$

Fecha de aceptación: 2019/06/30

Esta publicación se encuentra bajo licencia: Creative Commons ReconocimientoNoComercialSinObraDerivada 4.0 Internacional.

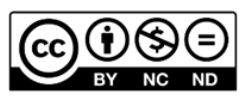

\section{Resumen}

El objetivo de a presente investigación es realizar un estudio para determinar el grado de acoso laboral o mobbing percibido en el personal docente de una Institución de educación superior (IES) pública, ubicada en la parte central del estado de Hidalgo, basada en la postura teórica y conceptual de Leymann. Esta investigación considera la conceptualización del fenómeno del mobbing, desde sus antecedentes, conceptos y estudios en el contexto organizacional. El instrumento que permite la medición del mobbing, sustentado con validez y confiabilidad es el cuestionario denominado LIPT-60 («Leymann Inventory of Psychological Terrorization»).

Los resultados obtenidos describen la existencia del mobbing en la institución de educación superior, con valores observados por debajo del nivel 1, específicamente en las subescalas de Desprestigio laboral, Incomunicación, e Intimidación manifiesta. Es por ello que en las instituciones de educación superior es fundamental realizar este tipo de trabajos con la finalidad de identificar, evaluar y conocer si existen riesgos de

\footnotetext{
${ }^{1}$ Dr. en Ciencias Administrativas, Universidad Autónoma del Estado de Hidalgo, México, enriquemarvar@gmail.com, https://orcid.org/0000-0001-6418-5292

${ }^{2}$ Dr. en Ciencias Administrativas, Universidad Autónoma del Estado de Hidalgo, México, explorerall@hotmail.com, https://orcid.org/0000-0003-0425-0800

${ }^{3}$ Maestro en Administración, Universidad Lasalle, México, Bernardino_martinez@uaeh.edu.mx,

${ }^{4}$ Maestro en Ciencias en Ingeniería de sistemas, Instituto Politécnico Nacional, México, ingjmh@uaeh.edu.mx,
} 
acoso laboral, para estar en posibilidad si así fuera el caso de disponer de medidas preventivas oportunas. En este contexto, el analizar los resultados permitirá establecer estrategias de prevención para disminuir o erradicar el acoso laboral en esta organización.

Palabras claves: 1731/PC/TR Mobbing, 1881/TM/TR, Acoso Laboral 3445/PC/TE Instituciones de Educación Superior

\begin{abstract}
The objective of this research is to conduct a study to determine the degree of bullying perceived or perceived in the teaching staff of a public institution of higher education (IES), located in the central part of the state of Hidalgo, based on the theoretical position and conceptual of Leymann. This research considers the conceptualization of the mobbing phenomenon, from its antecedents, concepts and studies in the organizational context. The instrument that allows the measurement of mobbing, supported with validity and reliability, is the questionnaire called LIPT-60 ("Leymann Inventory of Psychological Terrorization").

The results obtained describe the existence of mobbing in the institution of higher education, with values observed below level 1, specifically in the subscales of job loss, incommunication, and overt intimidation. That is why in higher education institutions is essential to perform this type of work in order to identify, assess and know if there are risks of workplace harassment, to be in possibility if this were the case to have timely preventive measures. In this context, analyzing the results will establish prevention strategies to reduce or eradicate workplace harassment in this organization.
\end{abstract}

Keywords: 1731/PC/TR Mobbing, 1881/TM/TR, Labor Harassment 3445/PC/TE Higher Education Institutions

\title{
Introducción
}

Aunque el mobbing es un término que data de hace muchos años (Carvajal y Dávila, 2013), en el campo de estudio de la

administración de organizaciones es relativamente novedoso, el cual es utilizado para establecer una conducta de 
hostigamiento o violencia psicológica, quienes se ejerce por un tiempo prolongado por parte de uno o varios individuos sobre otro u otras en el lugar de trabajo.

Román (2018) define al mobbing o acoso laboral cómo aquel comportamiento conductual nocivo por parte de un agresor con el objetivo de mancillar la dignidad y la estima de su víctima, valiéndose para ello de acciones denigrantes en el plano psicológico y emocional. En la administración moderna el capital humano es considerado uno de los factores más importante en las organizaciones incluso como factor determinante del éxito a fracaso de una organización. Una de las actividades principales de las organizaciones se encuentra el desarrollo integral de los miembros de la organización.

El presente estudio tiene como objetivo general conocer, diagnosticar y comprender los diversos fenómenos que afectan a las organizaciones, específicamente lo referente al acoso laboral o mobbing en los docentes de una institución de educación superior en el estado de Hidalgo, con la finalidad de obtener resultados que nos indiquen que factores son los que más inciden en las conductas de acosador hacia los docentes. En este contexto es importante destacar que la administración de organizaciones, de este siglo no ha conseguido encontrar con una solución a los problemas relacionados con las conductas negativas de personas que laboran en ellas. Este tipo de problema se le denomina mobbing.
El mobbing es un fenómeno que siempre ha existido en las organizaciones, en diversos grados y manifestaciones, y no es hasta hace un par de décadas que se inició como un tema de investigación científica en el ámbito de la psicología y por supuesto en la disciplina de la administración de organizaciones, por lo cual es importante destacar de manera puntual a lo concerniente al factor humano dentro de las organizaciones.

Aunque el estudio del mobbing se ha realizado en países desarrollados y en grandes empresas, en México existe pocos estudios, entre ellos se encuentra el modelo de diagnóstico de mobbing en las organizaciones mexicanas propuesto por Trujillo, Valderrábano y Hernández (2007) del Instituto Politécnico Nacional y la estimación de prevalencia del mobbing en los empleados de un Centro Universitario de Ciencias de la Salud llevado a cabo por Acosta, Pando, Aranda, y Aldrete (2005) de la Universidad de Guadalajara.

Los resultados de estas investigaciones se comprueban que la información es ciertamente escasa, por lo que a nivel nacional resulta muy baja la investigación que se ha realizado y los trabajos aún es ignorado por muchos. Este tipo investigaciones y factores que afectan a las organizaciones puede percibirse como un problema nocivo que afecta a los ambientes laborales de nuestro país y en creemos que también en Hidalgo.

Los diversos estudios reconocen que el fenómeno del mobbing aparece en cualquier organización, sin importar ni su tipo ni su tamaño. Las instituciones de 
educación no escapan a este fenómeno y conforme a los antecedentes encontrados el mobbing se manifiesta en cualquier espacio laboral, pero suele negarse, confundirse y/o enmascararse bajo otras denominaciones.

Es importante destacar que el mobbing aparece en cualquier organización sin importar su tamaño, ni su tipo. Es por ello por lo que la Instituciones de educación superior no escapan a este fenómeno. En este contexto el principal propósito es que las organizaciones deben de diagnosticar las causas que dan origen a este fenómeno, conocerlo y evitar su intensificación, para intervenir de forma inmediata y oportuna y sobre todo impedir que pueda convertirse en un elemento potencial para la presencia de este fenómeno de acoso en el ámbito laboral.

\section{Contexto teórico}

Hoy en día, existen diversas maneras o definiciones que explican el significado del fenómeno del mobbing, por lo cual es importante conceptualizar sus orígenes para conocer sus definiciones para obtener acepciones más claras de este fenómeno que afecta a las organizaciones. Los inicios del término "mobbing" tienen origen en el año 1966, cuando el etólogo Konrad Lorenz observó el comportamiento que tenían los animales y se percató de cómo un grupo de animales hostigaban a otro miembro de su misma manada, pero no fue hasta la década de los ochenta, cuando el psicólogo Heinz Leymann (1996) utiliza el término "mobbing" para referirse al acoso moral en el trabajo, por tanto, a este último se le considera cómo el primer estudioso en ofrecer una definición más asertiva acerca de este fenómeno (Fuentes, 2004).

De acuerdo con Leyman y Gustafsson este problema se estudió en 1984, cuando se publicó el informe del National Board of Ocupational Safety and Health de Estocolmo. La palabra mobbing se deriva del término en inglés "mob" derivada del latín mobile vulgus. El verbo inglés "to mob", se traduciría como: atacar en masa a alguien, acosar, atropellar; el sustantivo mob se distingue como: multitud, gentío, turba o muchedumbre. Mobbing vendría a ser una multitud que asedia a alguien.

Hirigoyen (1999) menciona que el "mobbing" son gestos, palabras, comportamientos, actitudes...) que atenta, por su repetición y sistematicidad, a la dignidad o a la integridad psíquica o física de una persona, poniendo en peligro su empleo o degradando el clima de trabajo. La OIT lo define como "la acción verbal o psicológica de índole sistemática, repetida o persistente por la que, en el lugar de trabajo o en conexión con el trabajo, una persona o un grupo de personas hiere a una víctima, la humilla, ofende o amedrenta" (Lugo, 2017).

González (2001), menciona que el Mobbing laboral puede considerarse como una forma de estrés psicosocial crónico, constituida por la acumulación prolongada de micro-trauma interpersonal repetidos que tienen lugar en el medio laboral.

Los autores Moreno, Garrosa, Galindo, San Julián, Rodríguez, Morante, y Losada, (2002), mencionan que el hostigamiento o acoso laboral (también conocido como mobbing) es un comportamiento (proceso) 
negativo entre superiores e inferiores jerárquicos de una organización laboral, a causa del cual el/la afectado/a es sometido a acoso y ataques sistemáticos durante mucho tiempo y de manera continuada de modo directo o indirecto - por parte de una o más personas. Alude a la violencia psíquica y, con regularidad, el objetivo es anular totalmente a su víctima.

El Diccionario de la Real Academia Española recoge el acoso moral como "la práctica ejercida en las relaciones personales, especialmente en el ámbito laboral, consistente en un trato vejatorio y descalificador hacia una persona, con el fin de desestabilizarla psíquicamente" . Como menciona el profesor Gasco (2011) "todas las definiciones inciden en el mismo conjunto de elementos: el maltrato continuado y deliberado, que puede ocasionar daños físicos, psíquicos e incluso patrimoniales y que persigue expulsar al sujeto de su situación laboral, creando un entorno intimidatorio, humillante $\mathrm{u}$ ofensivo que atenta contra la dignidad del trabajador".

Varias investigaciones desarrolladas han producido o han sido producto de la necesidad desarrollar $\mathrm{o}$ construir instrumentos que permitan evaluar el mobbing, tanto su presencia, como su grado e intensidad. Este fenómeno históricamente, se ha medido utilizando como unidad de análisis la percepción del sujeto y empleando generalmente la metodología propuesta por Leymann (1990).

Los resultados obtenidos no indican que la característica principal de la violencia es ser deliberada y comportar una actividad censurable. Con el tiempo y en diferentes espacios su carácter ha cambiado de la agresión física a los daños psíquicos, emocionales, económicos a través de amenazas u ofensas. En una institución púbica, algunos ejes que articulan la violencia son la distribución desigual de recursos o competencias, la vulnerabilidad (con respecto a las expectativas) y el control de la información entre diferentes individuos o grupos (Muñoz, 2003: 1218).

El estudio realizado en académicos de la Universidad de Ciencias y Artes de Chiapas, México Pérez, Sosa y Moreno (2008), arrojó que la violencia recibida presenta un porcentaje muy alto y muestra que han sido objeto de violencia psicológica en el trabajo por parte de sus superiores.

Los autores mencionan que "en correspondencia con algunas investigaciones que se han realizado para identificar el mobbing en académicos, los resultados obtenidos confirman que son las mujeres las que con mayor frecuencia son víctimas del acoso psicológico en el trabajo".

Martínez, E. (2018) menciona que para saber si un determinado comportamiento es una manifestación de mobbing, es necesario identificar y analizar las características y contextos o escenarios donde se presenta. Otra investigación efectuada a los docentes de una institución de educación superior en el estado de Guerrero, México (Méndez, Trejo y Rodríguez, 2010), menciona que el mobbing es un fenómeno que está presente en todas las organizaciones actuales y 
puede tener su origen en la conformación burocrática que caracteriza sobre todo a organismos públicos y la escasez del trabajo.

En su investigación hacen constatar la existencia del mobbing en los docentes que están expuestos al fenómeno y que se sienten afectados en diferentes grados por las diversas manifestaciones, comportamientos y estrategias de los cuales han sido objeto en su sitio de trabajo.

Fernández y Garnique (2014), argumentan que hay escuelas de educación superior que no han incluido ningún tipo de reglamentación acerca del acoso en cualquiera de sus manifestaciones. Aunque esta ausencia no indica una conducta admitida o ignorada, tampoco sugiere su existencia.

En la investigación realizada (Del Pino, 2014), se detectó una prevalencia mayor en la violencia psicológica en el trabajo en el personal docente evaluado en universidades de México; desde la perspectiva de género se identificaron diferencias en estas variables de estudio, cuyas puntuaciones más altas se presentaron en el personal femenino docente. Ante tal panorama, puede decirse que las profesoras universitarias se erigen en un grupo de alto riesgo laboral de presentar mayores problemáticas al respecto.

Estos hallazgos podrían estar proyectando la posible presencia de una cultura de violencia laboral de naturaleza emocional en las universidades. En un estudio realizado por Morales (2017), a maestros de los municipios de Juana Díaz y Ponce, en Puerto Rico, mostró que los maestros han sido acosados no solo por sus compañeros de trabajo, sino también por sus jefes inmediatos (directores escolares), e incluso han sufrido acoso de altos funcionarios, estos últimos jerárquicamente más poderosos que sus directores escolares.

Muñoz, López-Chau \& González (2018) en un estudio sobre la relación entre mobbing y la satisfacción laboral en docentes de instituciones de educación superior en México, encontraron que si existe dependencia estadística significativa entre estas dos variables, comprobaron la existencia de dependencia entre las dimensiones mobbing personal, desvalorización del trabajo, mobbing organizacional y sobrecarga de trabajo, con las dimensiones de satisfacción laboral: autorrealización, desempeño profesional, liderazgo y ambiente laboral.

\section{Materiales y métodos}

La presente investigación se considera descriptivo y cuantitativo, el tipo de estudio es no experimental y acorde a la evolución del fenómeno de estudio se define transversal y conforme a su orientación se clasifica investigación aplicada. La población observada fue de 95 docentes, por lo que se decidió encuestar a toda la población objetivo, para ello los docentes seleccionados fueron contactados en su lugar de trabajo e invitados a responder el cuestionario, además de realizar las 95 encuestas aplicadas de forma de auto respuesta, consistente en pedir al sujeto responsa 
directamente a las preguntas en la escala de Likert.

Todos los participantes respondieron a un cuestionario el cual considera datos sociodemográficos y sociolaborales, considerados en la adaptación española del LIPT, el LIPT-60 (González de Rivera y Rodríguez-Abuín, 2005).

El LIPT («Leymann Inventory of Psychological Terrorization»), (Leymann, 1990) es uno de los instrumentos más utilizados a nivel internacional para valorar el acoso

psicológico en el lugar de trabajo (González de Rivera \& Rodríguez-Abuín, 2005). El LIPT-60 es la versión modificada y traducida al castellano del LIPT dicotómico original (de cuarenta y cinco ítems).

Tratándose de un cuestionario escalar autoadministrado que objetiva y valora sesenta diferentes estrategias de acoso psicológico a través de una escala tipo Likert sobre la intensidad con que cada trabajador percibe dichas conductas en su lugar de trabajo, desde cero (la conducta no ha tenido lugar) hasta cuatro (conducta de intensidad máxima). La adaptación española goza de buenas propiedades psicométricas y se encuentra desde 2005 (González de Rivera \& Rodríguez-Abuín, 2005).

\section{Resultados}

Para la realización del estudio se consideró a una institución de educación superior (IES) ubicada en el estado de Hidalgo, México, con una antigüedad desde su creación de 15 años y representativa de educación superior pública de la región centro y sureste del estado de Hidalgo y participaron 95 docentes de dicha institución.

El diseño de la presente investigación desde una perspectiva cuantitativa, se realizó para describir el concepto de mobbing, definición y conceptualización en el ámbito educativo, precisamente en el lugar de trabajo de los docentes en la institución educativa

Tabla 1. Género Docente

\begin{tabular}{lrrr}
\hline Genero & Frecuencia & Porcentaje & $\begin{array}{c}\text { Porcentaje } \\
\text { Acumulado }\end{array}$ \\
\hline Masculino & 70 & $73,7 \%$ & $73,7 \%$ \\
Femenino & 25 & $26,3 \%$ & $100,0 \%$ \\
\hline Total & 95 & $100,0 \%$ & $100,0 \%$ \\
\hline
\end{tabular}

Fuente. Desarrollo propio

En la Tabla 1 los valores más representativos de trabajadores encuestados, a destacar el $73.7 \%$ son del género masculina, mientras que el $26.3 \%$ son del género femenino, se observa una falta de equidad laboral.

Tabla 2. "Edad del docente"

\begin{tabular}{lccc}
\hline Validos & Frecuencia & Porcentaje & $\begin{array}{c}\text { Porcentaje } \\
\text { acumulado }\end{array}$ \\
\hline 24 a 29 años & 45 & 47.4 & 47.4 \\
30 a 35 & 8 & 8.4 & 55.8 \\
42 a 47 & 18 & 18.9 & 74.7 \\
48 a 53 & 3 & 3.2 & 77.9 \\
54 a 59 & 7 & 7.4 & 85.3 \\
60 a más & 14 & 14.7 & 100.0 \\
\hline Total & & 95 & 100.0 \\
\hline Fuente: Desarrollo propio & &
\end{tabular}

En la Tabla 2 se observan los valores más representativos de la edad de los docentes encuestados, a destacar el $47.4 \%$ tiene una edad de 24 a29 años, mientras que el 18.9 
$\%$ son de 48 a 53 años, y finalmente el $14.7 \%$ representa una edad de 60 año a más

Tabla 3. "Antigüedad del Docente"

\begin{tabular}{lccc}
\hline Validos & Frecuencia & Porcentaje & $\begin{array}{c}\text { Porcentaje } \\
\text { acumulado }\end{array}$ \\
\hline 1-2 años & 33 & 34.7 & 34.7 \\
03-abr & 19 & 20.0 & 54.7 \\
05-jun & 0 & 0 & 54.7 \\
07-ago & 18 & 18.9 & 73.7 \\
09-oct & 0 & 0 & 73.7 \\
11-dic & 1 & 1.1 & 74.7 \\
13-14 & 22 & 23.2 & 97.9 \\
15-más años & 2 & 2.1 & 100 \\
\hline Total & 95 & 100.0 & \\
\hline
\end{tabular}

Fuente: Desarrollo propio

En la Tabla 3, el $34.7 \%$ del total de docentes encuestados cuentan una antigüedad laboral entre 1- 2 años, mientras que el $20 \%$ representa una antigüedad de 3 a 4 años, lo que nos indica que el personal dura muy poco en su trabajo.

\section{1.- Subescala de índice de desprestigio laboral (DL).}

Respecto al Desprestigio en el trabajo, distorsión de la comunicación, calumnias o medidas restrictivas, variables consideradas en el instrumento de medición, se observa un promedio obtenido de 0.73 en la siguiente gráfica.

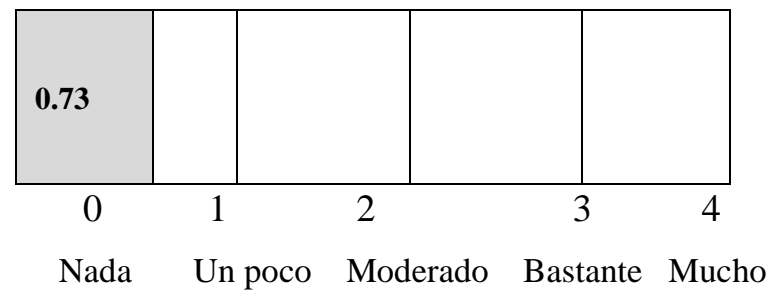

El factor de 0.73 nos indica que el fenómeno se percibe y que existen practicas dirigidas al desprestigio del trabajo, distorsión de la comunicación y medidas restrictivas.

Generalmente aplicada a docentes de menor antigüedad en el trabajo y a aquellos que difieren en apreciaciones de los directivos de la institución, debido a que consideran que la forma de imponer autoridad, es indicar a los docentes lo que es pertinente sin dar explicaciones concisas $o$ sustentadas bajo alguna normatividad y enfatizando la importancia que existen en los grupos de poder en la institución.

\section{2.- Subescala de entorpecimiento del progreso (EP),}

La subescala del entorpecimiento al progreso referente al bloqueo sistemático de la actividad laboral, degradando al trabajo con tarea inapropiadas en la forma o contenido, de acuerdo con las competencias, consideradas en el instrumento de medición, y la valoración del instrumento de medición se observa en la siguiente gráfica.

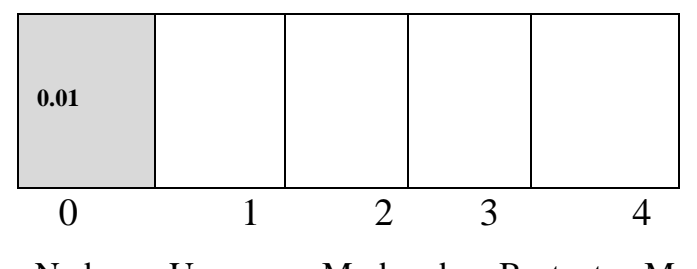

Nada Un poco Moderado Bastante Mucho

El factor de 0.01 nos indica que el fenómeno que se percibe es casi nulo, denotando algunas prácticas dirigidas al entorpecimiento del progreso generalmente observado en a docentes de nuevo ingreso o menor antigüedad en el trabajo debido a factores de competencia por obtener mejores y más hora de clase. 
3.- Subescala de incomunicación o bloqueo de la comunicación (BC)

El factor de 0.26 indica que el fenómeno se percibe y existe un poco, denotando algunas prácticas dirigidas al bloqueo de la comunicación dentro de la organización y fuera de la organización.

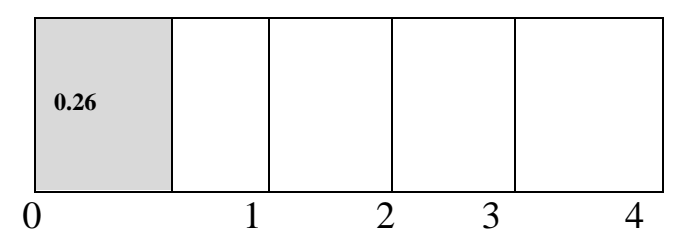

Nada Un poco Moderado Bastante Mucho

Estas circunstancias se originan por la falta de personal administrativo dentro de la institución y otra por la ubicación física de la institución y el lugar donde se imparte la docencia, y las oficinas administrativas, existiendo distancias considerables entre los departamentos y las aulas de docencia por lo cual pueden ser factores que inciden en bloqueo de la comunicación entre docentes.

\section{4.- Subescala de intimidación} encubierta (IE)

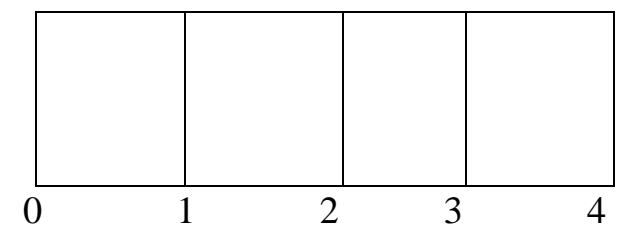

Nada Un poco Moderado Bastante Mucho

El factor de 0.indica que el fenómeno no se percibe intimidación encubierta.

\section{5.- Subescala de intimidación} manifiesta (IM)

Respecto a la intimidad manifiesta, en lo que se refiere a amenazas o restricciones que se impone en forma directa, no disimula incluso en público, tales como amenazas verbales, gritos o poner en ridículo variables consideradas en el instrumento de medición, y la valoración del instrumento de medición se observa en la siguiente gráfica.

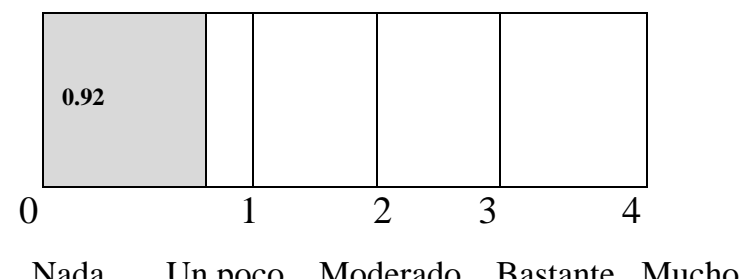

El factor de 0.92 nos indica que el fenómeno existe practicas dirigidas a la amenazas $\mathrm{o}$ intimidaciones $\mathrm{y}$ medidas restrictivas generalmente aplicada a la mayoría de docentes, dentro de la cuales se encuentran la negación de permisos para ausentarse por motivos personales, así como también la negación del uso de los días de permiso con goce de sueldo, esto si dar explicación alguna y sin duda alguna son utilizadas como medidas de control del personal y abusando del estilo de dirección autócrata que en la presente administración estatal, se encuentra en todas las áreas.

\section{6.- Subescala de desprestigio} personal (DP)

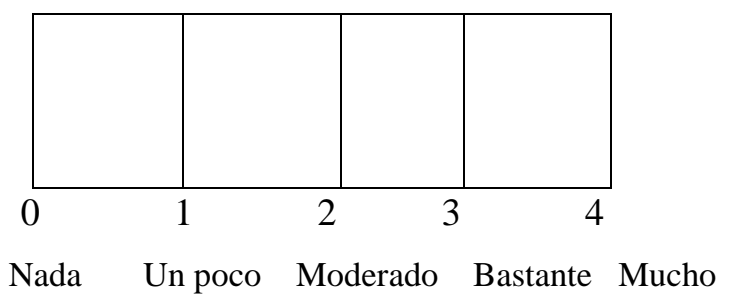

El factor de 0 indica que el fenómeno no se percibe desprestigio personal.

\section{Discusión}

Considerando las teorías y conceptos se infiere que el mobbing es un fenómeno 
que está presente en las organizaciones actuales y puede tener su origen es diversos fenómenos dimensionales entre los que destacan: La falta d espacios laborales, las políticas públicas al vapor enfocadas al ahorro y despido de personal, La burocracia existente y anquilosadas históricamente en las instituciones de educación superior públicas en Hidalgo.

El análisis de las diferentes variables estudiadas nos permite explicar cuál es la respuesta humana a dicho fenómeno, el cual se logró al estudiar con detenimiento y las relaciones que estadísticamente se obtuvieron a través del análisis de datos y al captar en forma personal los comentarios e inquietudes de los trabajadores al momento de realizar el estudio de campo, obteniéndose como resultado la presencia del mobbing en docentes de una institución de educación superior en Hidalgo en las subescalas de: Desprestigio laboral (DL), Entorpecimiento del progreso(EP), Incomunicación o bloqueo de la comunicación $\quad(\mathrm{BC}), \quad$ Intimidación manifiesta (IM), cuyos valores se encuentran dentro de 0 y 1 indicando nada y un poco.

Es importante denotar los resultados estadísticos qué si bien son resultados fríos, nos indican que situación se presenta en los lugares de trabajo. En este contexto se pudo observar que el mobbing es un factor psicosocial que se encuentra presente en las organizaciones actuales.

\section{Conclusiones}

Se concluye que existe mobbing en la institución de educación superior (IES) en el estado de Hidalgo y producto de algunos factores que pueden incidir en esta institución, entre ellos, la falta de perfil directivo de quienes dirigen a las instituciones educativas en el estado de Hidalgo, aunado a comportamientos autocráticos y dirección baja amenazas, además de la conformación burocrática en los organismos públicos y la falta de humanización como elemento fundamental en la dirección de las instituciones. En este contexto fue posible constatar que aquellos docentes que están expuestos al fenómeno y que se siente afectados en diferente grado las diversas manifestaciones, comportamientos y estrategias de las cuales han sido objeto en su centro de trabajo.

La presente investigación ayuda el diagnóstico y conocimiento y presencia del mobbing en instituciones de educación superior en el estado de Hidalgo, los resultados de esta investigación pueden anticipar la aparición, propagación y factores que puedan originar el mobbing y que puedan afectar al quehacer laboral en estas importantes instituciones educativas en Hidalgo, y estar en posibilidad de establecer decisiones para el establecimiento de estrategias de prevención y reducción del mobbing y disminuir su impacto en los docentes.

\section{Referencias bibliográficas}

Acosta, M., Pando, M., Aranda, C. y Aldrete, M.G. (2005). El acoso moral en el trabajo en los empleados del Centro Universitario de Ciencias de la Salud de la Universidad de Guadalajara. Investigación en Salud, 7(1), 16-23.Recuperado de: 
https://www.medigraphic.com/pdfs/invsal /isg-2005/isg051d.pdf

Del Pino, R. (2014). Análisis diferencial de la violencia y el acoso psicológico en el trabajo del personal docente: génesis de estudio para una gestión educativa contra el mobbing universitario desde una perspectiva de género. Recuperado de http://congreso.investiga.fca.unam.mx/do cs/xix/docs/13.02.pdf

González de Rivera, JL y RodríguezAbuín, MJ. (2005). Cuestionario de estrategias de acoso en el trabajo. El LIPT60. Editorial EOS, Madrid, 2005.

González, J.L. (2001). Nosología psiquiátrica del estrés. Psiquis. Revista de psiquiatría, psicología médica y psicosomática, 22(1), 9-15.

Fernández, S. y Garnique, F. (2014). Acoso laboral, mobbing: análisis, propuestas y recomendaciones para instituciones de educación superior. Revista Gestión y Estrategia, 1(45), 81-94. Recuperado

de http://gestionyestrategia.azc.uam.mx/inde x.php/rge/article/view/582

Fuentes, J.C. (2004). Mobbing Acoso Laboral Psicoterrorismo en el trabajo. España: Arán Ediciones.

Gasco, E. (2011). Acoso moral laboral o mobbing. Recuperado de https://librosrevistas-derecho.vlex.es/vid/acoso-morallaboral-mobbing-461454594

Hernández, T. y Carrión, A. (2018). Mobbing un factor de riesgo laboral en México. Principales aspectos teóricos y hallazgos de investigación. México: Miguel Ángel Porrúa.
Hirigoyen, M.F. (1999). El acoso moral. El maltrato psicológico en la vida cotidiana. Barcelona: Paidós.

Leymann, H. (1990). Mobbing and psychological terror at workplaces. Violence and victims, 5(2), 119-126. Doi: 10.1891/0886-6708.5.2.119

Leymann, H. (1996). The content and development of mobbing at work. European Journal of Work and Organizational Psychology, 5(2), 165184. Recuperado de http://www.organizedmobbing.com/Leym ann1996.pdf

Lugo, M. (2017). Acoso Laboral Mobbing. Recuperado de: http://appweb.cndh.org.mx/biblioteca/arc hivos/pdfs/Acoso-Laboral-Mobbing.pdf

Martínez, E. (2018), Características y escenarios principales de acoso en el trabajo. En Hernández, T. y Carrión, A. (coords.), Mobbing un factor de riesgo laboral en México. Principales aspectos teóricos y hallazgos de investigación (págs. 15-26). México: Miguel Ángel Porrúa.

Méndez, I., Trejo, M. y Rodríguez, F. (2010). Estudio para la determinación del grado de acoso laboral (mobbing) de los trabajadores de una institución de educación superior en el estado de Guerrero. Recuperado de: http://acacia.org.mx/busqueda/pdf/12_11 _acoso_laboral.pdf

Morales, J. (2017). Acoso psicológico laboral (moobing) y su impacto en el desempeño laboral. Aplicación a los maestros de los municipios de Juana Díaz y Ponce, Puerto Rico. Recuperado de 
https://dialnet.unirioja.es/servlet/tesis?cod igo $=68328$

Moreno, B., Garrosa, E., Galindo, C., San Julián, L., Rodríguez, R., Morante, M. E., y Losada, M. (2002). Manual sobre acoso psicológico. Recuperado de: http://www.fespugtmadrid.es/wpcontent/uploads/saludlaboral/documentacion/manuales $\% 20 \mathrm{de} \%$ 20prevencion/MANUAL\%20DE\%20AC OSO\%20PSICOLOGICO.pdf.

Muñoz, C. J. P., López-Chau, A., \& González, P. M. (2018). Estudio sobre la relación entre mobbing y la satisfacción laboral en docentes de instituciones de educación superior en México. European Scientific Journal, 14(4), 298-314.

Muñoz, Antonio (2003). "Tras las huellas de la violencia”. En José Manuel Martín Morillas, Los sentidos de la violencia, Granada, Universidad de Granada.
Pérez, O.C., Sosa, M. y Moreno, M.P. (2008). Mobbing y estrés en académicos de la Universidad de Ciencias y Artes de Chiapas. Tesis doctoral Universidad de Ciencias y Artes de Chiapas). Recuperado de

http://www.factorespsicosociales.com/seg undoforo/simposio/cruz-ovalle-pando.pdf citas bibliográficas-bases de datos uta.

Román, J.G. (2018). Mobbing. Estudio realizado en el personal docente $\mathrm{y}$ administrativo de las Facultades 1 y 2 de la Universidad de Cuenca. Recuperado de: http://dspace.ucuenca.edu.ec/handle/1234 56789/29720

Trujillo, M.M., Valderrábano, M.L. y Hernández, R. (2007).

Mobbing: historia, causas, efectos $\mathrm{y}$ propuesta de un modelo para las organizaciones mexicanas. Revista Innovar, 17(29), 71-92. 